\title{
Serum Vitamin D Levels in Patients with Myelodysplastic Syndromes: A Retrospective Single-Center Analysis
}

\author{
Catharina Müller-Thomas ${ }^{a}$ Heinz Tüchler ${ }^{b}$ Martina Rudelius ${ }^{c}$ \\ Heike Schneider $^{d}$ Sabrina Pfefferkorn ${ }^{a}$ Katharina S. Götze ${ }^{\text {a, e }}$ \\ ${ }^{a}$ Department of Medicine III, Hematology and Oncology, Technische Universität München, Munich, Germany; \\ b Ludwig Boltzmann Institute for Leukemia Research, Vienna, Austria; ' Institute of Pathology, \\ Ludwig-Maximilians-Universität, Munich, Germany; ${ }^{\mathrm{d}}$ Institute of Clinical Chemistry und Pathobiochemistry, \\ Technische Universität München, Munich, Germany; ${ }^{e}$ German Cancer Consortium (DKTK) and German Cancer \\ Research Center (DKFZ), Heidelberg, Germany
}

\section{Keywords}

Myelodysplastic syndromes · Serum vitamin D level ·

Association between vitamin D and MDS

\begin{abstract}
Background/Aims: There is growing evidence supporting the role of innate immune deregulation and inflammation in the pathogenesis of myelodysplastic syndromes (MDS). Vita$\min \mathrm{D}(\mathrm{VD})$ is known to be involved in various immune and epigenetic processes. This analysis aimed to evaluate serum VD levels in patients with MDS and to analyze associations between serum VD levels and disease characteristics. Methods: Serum levels of 25-hydroxyvitamin D3 (25(OH)-D3), the major form of VD in human serum, were measured by chemiluminescence immunoassay in 62 unselected patients with MDS. Associations between serum 25(OH)-D3 levels and disease characteristics were analyzed using Kendall's tau and two-sided $p$ values. Results: The median serum $25(\mathrm{OH})$-D3 level was markedly reduced $(17.5 \mathrm{ng} / \mathrm{mL})$. Patients with lower-risk disease features had lower serum 25(OH)-D3 levels than patients with higher-risk disease features with regard to medullary blast counts ( $16 \mathrm{vs.} 31 \mathrm{ng} / \mathrm{mL}$,
\end{abstract}

(C) 2019 S. Karger AG, Basel

\section{E-Mail karger@karger.com www.karger.com/aha \\ KARGER}

$p<0.001)$, the revised international prognostic scoring system (13 vs. $30.5 \mathrm{ng} / \mathrm{mL}, p=0.001$ ), and blood counts. Conclusions: We show that patients with lower-risk disease characteristics exhibit lower serum VD levels than patients with higher-risk disease characteristics. Whether these findings might reflect innate immune deregulation has to be investigated in further studies.

c) 2019 S. Karger AG, Basel

\section{Introduction}

Myelodysplastic syndromes (MDS) represent a group of dysplastic bone marrow disorders, typically accompanied by peripheral cytopenia due to ineffective hematopoiesis with a variable risk of transformation into secondary acute myeloid leukemia. The pathogenesis of MDS is considered as multifactorial. In recent years, hematopoietic stem and progenitor cell alterations caused by genetically and epigenetically dysregulated genes have been identified [1-5]. Furthermore, the role of the innate immune system in the development of MDS has been elucidated $[1,6]$. It has been shown that chronic suppressive 
inflammation mediated by expansion of myeloid-derived suppressor cells in the bone marrow of MDS patients contributes to myeloid lineage skewing and ineffective hematopoiesis [7]. In addition, mesenchymal stem cells have gained growing interest regarding their putative contribution to the pathogenesis of MDS, their strong immunomodulatory capabilities, and their tremendous therapeutic potential for autoimmune and inflammatory diseases [8].

Vitamin D (VD) is increasingly thought to play a role in regulating immunity $[9,10]$. Particularly, VD deficiency has been largely documented in various autoimmune diseases, such as autoimmune thrombocytopenia, rheumatoid arthritis, and inflammatory bowel diseases [11, 12]. Furthermore, VD has been attributed roles in the pathogenesis of cancer, mainly via VD receptor mediated epigenetic activity $[13,14]$.

The regulating activity of VD in immune and epigenetic processes - both key components of MDS pathogenesis - prompted us to: (a) assess serum levels of VD in a cohort of 62 unselected patients with MDS, (b) associate serum levels of VD with disease characteristics, and (c) investigate whether our findings might serve as a valuable basis for further and deeper investigations of the impact of VD on innate immune modulation in MDS.

\section{Patients and Methods}

\section{Patients}

This retrospective analysis included 62 unselected patients with MDS who were treated at the Department of Medicine III, Hematology and Oncology, Technical University Munich, Germany, between September 2015 and September 2017. Written informed consent in accordance with the Declaration of Helsinki was provided by all patients. The diagnosis of MDS was made according to the 2016 revision of the World Health Organization classification of myeloid neoplasms and acute leukemia [15]. MDS risk assessment was based on the revised international prognostic scoring system (IPSS-R) [16]. Karyotypes were classified according to the new comprehensive cytogenetic scoring system [17]. Blood samples for the analysis of serum level of 25-hydroxyvitamin D3 $(25(\mathrm{OH})-\mathrm{D} 3)$ and full blood cell count were obtained at the same time point as part of routine care. The assessment of serum levels of $25(\mathrm{OH})$-D3 was intended as real-world experience; thus, serum levels of 25(OH)-D3 were measured once in every patient regardless of the disease course (initial diagnosis, advanced disease) and treatment (untreated, best supportive care, disease-modifying agents).

\section{Clinical and Laboratory Parameters}

Clinical and laboratory data were collected from medical records. The neutrophil to lymphocyte ratio (NLR) was defined as the absolute neutrophil count (ANC) divided by absolute lymphocyte count. 25(OH)-D3 reflects the major form of VD in human
Table 1. Patients' demographic and clinical characteristics $(n=62)$
Age, years

Male/female, $n$

WHO 2016 classification

MDS-MLD/MDS-MLD-RS

MDS-EB1

MDS-EB2

IPSS-R

Very low/low

Intermediate

High/very high

Cytogenetics

Very good/good

Intermediate

Poor/very poor

Median ANC, $\times 10^{9} / \mathrm{L}$

ANC

$<0.5 \times 10^{9} / \mathrm{L}$

$0.5-0.99 \times 10^{9} / \mathrm{L}$

$\geq 1.0 \times 10^{9} / \mathrm{L}$

PLT, $\times 10^{9} / \mathrm{L}$

$\mathrm{Hb}, \mathrm{g} / \mathrm{dL}$

NLR

Therapy

IPSS-R very low/low

IPSS-R intermediate

IPSS-R very high/high
$71.3(38.3-89.1)$

$37 / 25$

$49(79)$

$5(8)$

$8(13)$

$33(53)$

$19(31)$

$10(16)$

$50(81)$

7 (11)

$5(8)$

$1.86(0.08-11.92)$

$7(11)$

$11(18)$

$44(71)$

$144(9-476)$

$9.4(6.1-15.7)$

$1.49(0.08-47.7)$

$11 / 33(33)$

$8 / 19(42)$

$2 / 10(20)$
Data are presented as the median (range) or $n(\%)$. WHO, World Health Organization; MDS, myelodysplastic syndromes; MLD, multilineage dysplasia; RS, ring sideroblasts; EB, excess blasts; IPSS-R, revised international prognostic scoring system; ANC, absolute neutrophil count; NLR, neutrophil to lymphocyte ratio.

serum; thus, total serum $25(\mathrm{OH})-\mathrm{D} 3$ is considered to be the best indicator of VD supply. VD was measured as $25(\mathrm{OH})-\mathrm{D} 3$ by a chemiluminescence immunoassay in serum (LIASON ${ }^{\circledR} 25 \mathrm{OH}$ TOTAL, DiaSorin Inc. USA). LIASON ${ }^{\circledR} 25 \mathrm{OH}$ TOTAL is a direct competitive immunoassay: following the release from the binding protein, total $25(\mathrm{OH})-\mathrm{D} 3$ is captured by a goat antibody coated on magnetic beads. After adding isoluminol-labeled 25(OH)-D3 and several incubation and wash steps, $25(\mathrm{OH})-\mathrm{D} 3$ is determined in inverse proportion to the signal.

\section{Statistical Analysis}

With the exception of counts and percentages for categorical variables, numerical variables were described by the median and range. Correlations between dichotomous, ordinal, and metric variables were quantified by Kendall's tau $(\tau)$ and tested by the related $p$ values. Two-sided $p$ values $<0.05$ were considered significant. Confidence intervals refer to $95 \%$ coverage. In line with the essentially exploratory nature of the study, no adjustment for multiple testing was applied. The statistical analysis was performed with the statistical environment $\mathrm{R}$ version 3.2.3 Patched (2015-1221 r69801) [18]. 
Table 2. Median serum levels of 25(OH)-D3 according to disease, IPSS-R, and cytogenetics

\begin{tabular}{lll}
\hline \multicolumn{2}{l}{ Median 25(OH)-D3, ng/mL } \\
\hline $\begin{array}{l}\text { All patients }(n=62) \\
\text { Disease }\end{array}$ & & \\
$\quad$ MDS $<5 \%$ blasts $(n=49)$ & 16 & $\tau=0.386$ (95\% CI 0.190 to 0.582) \\
$\quad$ MDS $\geq 5 \%$ blasts $(n=13)$ & 31 & $p<0.001$ \\
IPSS-R & 13 & $\tau=0.334$ (95\% CI 0.157 to 0.511) \\
$\quad$ Very low/low $(n=33)$ & 17 & $p=0.001$ \\
$\quad$ Intermediate $(n=19)$ & 30.5 & \\
$\quad$ High/very high $(n=10)$ & 17 & $\tau=0.092(95 \%$ CI -0.111 to 0.295$)$ \\
Cytogenetics & 21 & $p=0.380$ \\
$\quad$ Very good/good $(n=50)$ & 31 & \\
$\quad$ Intermediate $(n=7)$ & & \\
$\quad$ Poor/very poor $(n=5)$ & &
\end{tabular}

25(OH)-D3, 25-hydroxyvitamin D3; MDS, myelodysplastic syndromes; IPSS-R, revised international prognostic scoring system.

\section{Results}

A total of 62 patients with MDS were evaluated for this retrospective analysis. The patients' demographic and clinical characteristics are summarized in Table 1. The median age was 71.3 years (range 38.3-89.1). The cohort consisted of 37 males (60\%) and 25 females (40\%); the male to female ratio was 1.5. The majority of MDS patients $(n=49 ; 79 \%)$ had bone marrow blasts $<5 \%$. MDS risk assessment according to IPSS-R revealed very low/low risk in 33 patients (53\%), intermediate risk in 19 patients (31\%), and high/very high risk in 10 patients (16\%). Cytogenetics were favorable (very good, good) in 50 patients (81\%), intermediate in 7 patients $(11 \%)$, and adverse (poor, very poor) in 5 patients (8\%). The median ANC was $1.86 \times 10^{9} / \mathrm{L}$ (range $\left.0.08-11.92 \times 10^{9} / \mathrm{L}\right) ; 7$ patients $(11 \%)$ were deeply neutropenic with ANC $<0.5 \times 10^{9} / \mathrm{L}$ (median $\left.0.2 \times 10^{9} / \mathrm{L}\right), 11$ patients $(18 \%)$ had moderate neutropenia with ANC between 0.5 and $<1.0 \times 10^{9} / \mathrm{L}$ (median $0.66 \times$ $\left.10^{9} / \mathrm{L}\right)$, and 44 patients $(71 \%)$ showed ANC $\geq 1.0 \times 10^{9} / \mathrm{L}$ (median $\left.2.58 \times 10^{9} / \mathrm{L}\right)$. The median PLT was $144 \times 10^{9} / \mathrm{L}$ (range $9-476 \times 10^{9} / \mathrm{L}$ ) and median $\mathrm{Hb}$ level was $9.4 \mathrm{~g} / \mathrm{dL}$ (range 6.1-15.7). The median NLR was 1.49 (range 0.0847.7). At the time of VD assessment - and beyond best supportive care $-33 \%(n=11 / 33)$ of patients with very low/low IPSS-R obtained therapy (erythropoietin, $n=9$; lenalidomide, $n=1$; luspatercept, $n=1), 42 \%(n=8 / 19)$ of patients with intermediate IPSS-R (erythropoietin, $n=6$; azacitidine, $n=2)$, and $20 \%(n=2 / 10)$ patients with high/very high IPSS-R (azacitidine, $n=2$ ).

Analysis of Vitamin D in Patients with

Myelodysplastic Syndromes
The median serum level of 25(OH)-D3 of all patients was $17.5 \mathrm{ng} / \mathrm{mL}$ (Table 2). Patients with MDS with medullary blasts $<5 \%$ had a median serum level of $25(\mathrm{OH})-\mathrm{D} 3$ of $16 \mathrm{ng} / \mathrm{mL}$, whereas patients with MDS $\geq 5 \%$ medullary blasts exhibited a significantly higher median serum level of $25(\mathrm{OH})$-D 3 of $31 \mathrm{ng} / \mathrm{mL}$ ( $\tau=0.386,95 \%$ CI $0.190-0.582$, $p<0.001$; Table 2; Fig. 1a). According to IPSS-R the median serum level of $25(\mathrm{OH})-\mathrm{D} 3$ was 13,17 , and $30.5 \mathrm{ng} / \mathrm{mL}$ with very low/low, intermediate, and high/very high risk MDS, respectively $(\tau=0.334,95 \%$ CI $0.157-0.511, p=0.001$; Table 2 ; Fig. 1b). There was an increasing median serum level of $25(\mathrm{OH})-\mathrm{D} 3$ in respect to cytogenetics with 17,21 , and 31 $\mathrm{ng} / \mathrm{mL}$ in very low/low, intermediate, and high/very high risk patients but statistical significance was not reached ( $\tau=0.092,95 \%$ CI -0.111 to $0.295, p=0.380$; Table 2 ; Fig. $1 \mathrm{c}$ ).

Patients with an ANC $\geq 1.0,0.50-0.99$, and $<0.5 \times$ $10^{9} / \mathrm{L}$ exhibited a median serum level of $25(\mathrm{OH})-\mathrm{D} 3$ of 16 , 20 , and $29 \mathrm{ng} / \mathrm{mL}$, respectively ( $\tau=-0.277,95 \%$ CI -0.458 to $-0.096, p=0.008$; Table 3; Fig. 2a). In patients with NLR $\geq 1.49$ (median NLR) and NLR $<1.49$ the median serum level of $25(\mathrm{OH})-\mathrm{D} 3$ was 14 and $21 \mathrm{ng} / \mathrm{mL}$, respectively $(\tau=-0.228,95 \%$ CI -0.422 to $-0.033, p=0.033$; Table 3; Fig. 2b). Patients with NLR $\geq 3$ and NLR $<3$ showed a median serum level of 25(OH)-D3 of 13.5 and $20 \mathrm{ng} / \mathrm{mL}$, respectively $(\tau=-0.212,95 \%$ CI -0.409 to $-0.015, p=0.048$; Table 3; Fig. 2b). Patients with a PLT $\geq 50 \times 10^{9} / \mathrm{L}$ attained a median serum level of $25(\mathrm{OH})-\mathrm{D} 3$ of $16 \mathrm{ng} / \mathrm{mL}$, while in patients with a PLT $<50 \times 10^{9} / \mathrm{L}$ the median serum level of 25(OH)-D3 was $29 \mathrm{ng} / \mathrm{mL}(\tau=$ $-0.192,95 \%$ CI -0.412 to $0.027, p=0.072$; Table 3; Fig. 2 c) . 


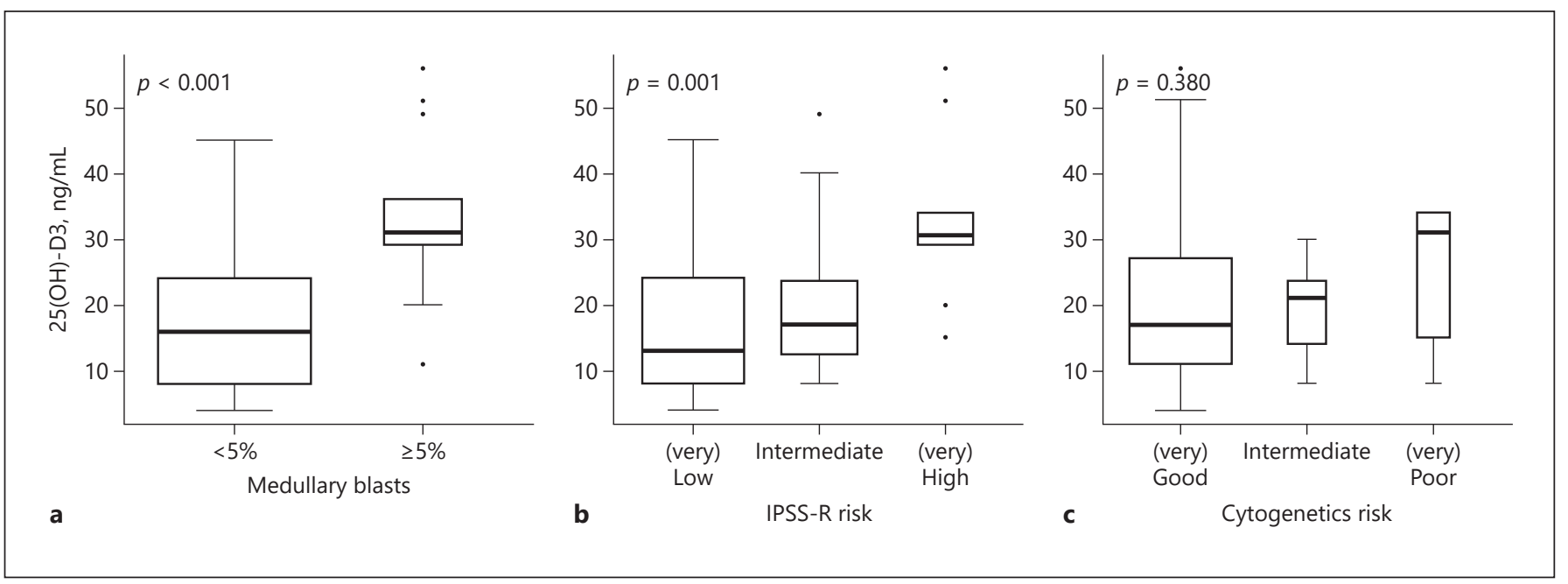

Fig. 1. Median serum levels of 25(OH)-D3 in patients considering medullary blasts (a), IPSS-R MDS risk (b), and cytogenetics $(\mathbf{c})$.

Table 3. Median serum levels of 25(OH)-D3 according to ANC, NLR, and PLT

\begin{tabular}{lll}
\hline \multicolumn{2}{l}{ Median $25(\mathrm{OH})-\mathrm{D} 3, \mathrm{ng} / \mathrm{mL}$} \\
\hline All patients $(n=62)$ & 17.5 & \\
ANC & 16 & $\tau=-0.277(95 \% \mathrm{CI}-0.458$ to -0.096$)$ \\
$\quad \geq 1.0 \times 10^{9} / \mathrm{L}(n=44)$ & 20 & $p=0.008$ \\
$\quad 0.5-0.99 \times 10^{9} / \mathrm{L}(n=11)$ & 29 & \\
$\quad<0.5 \times 10^{9} / \mathrm{L}(n=7)$ & 14 & $\tau=-0.228(95 \% \mathrm{CI}-0.422$ to -0.033$)$ \\
NLR & 21 & $p=0.033$ \\
$\quad \geq 1.49(n=31)$ & 13.5 & $\tau=-0.212(95 \% \mathrm{CI}-0.409$ to -0.015$)$ \\
$\quad<1.49(n=31)$ & 20 & $p=0.048$ \\
$\quad \geq 3(n=14)$ & 16 & $\tau=-0.192(95 \% \mathrm{CI}-0.412$ to 0.027$)$ \\
$\quad<3(n=48)$ & 29 & $p=0.072$ \\
\hline PLT & & \\
$\quad<50 \times 10^{9} / \mathrm{L}(n=51)$ & & \\
\hline
\end{tabular}

25(OH)-D3, 25-hydroxyvitamin D3; ANC, absolute neutrophil count; NLR, neutrophil to lymphocyte ratio.

\section{Discussion}

VD is a pleiotropic hormone of the steroid/thyroid superfamily, classically known for calcium homeostasis and bone health. In recent years, it has become clear that VD exerts many extraskeletal effects, ranging from immune modulation to cell differentiation.

There is general agreement that plasma or serum 25(OH)-D3 concentration represents total VD from exposure to both ultraviolet irradiation and dietary sources and can be used as a reliable biomarker of VD status [19].
However, the optimal target value of serum 25(OH)-D3 concentration has not been established, and there is substantial debate about this issue. The Endocrine Society favors maintaining the blood level of 25(OH)-D3 above $30 \mathrm{ng} / \mathrm{mL}$ (75 nmol/L) [20]. Accordingly, VD insufficiency is defined as a $25(\mathrm{OH})-\mathrm{D} 3$ concentration of $21-29 \mathrm{ng} /$ $\mathrm{mL}$, and VD deficiency is defined as a $25(\mathrm{OH})-\mathrm{D} 3$ concentration of less than $20 \mathrm{ng} / \mathrm{mL}$ [20]. Regarding our cohort of 62 patients with MDS, median serum levels of $25(\mathrm{OH})$-D3 were $17.5 \mathrm{ng} / \mathrm{mL}$, revealing VD deficiency in a substantial subgroup of the cohort. 


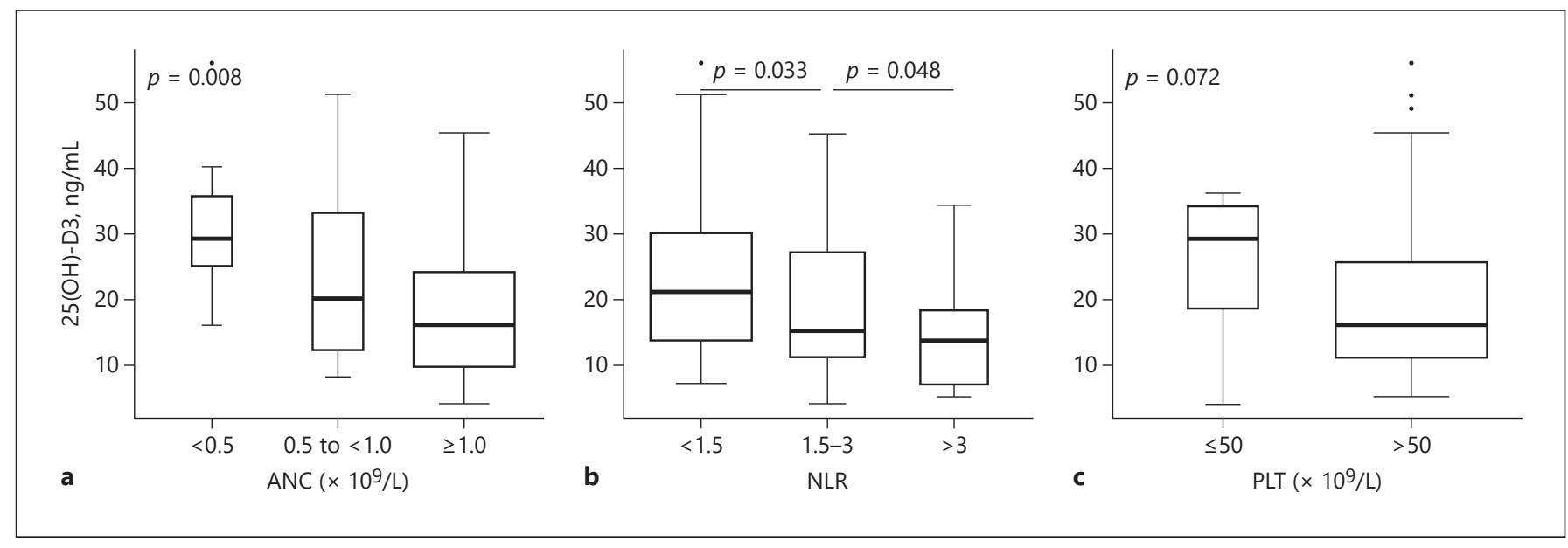

Fig. 2. Median serum levels of 25(OH)-D3 in patients considering ANC (a), NLR (b), PLT (c).

Interestingly, we found serum levels of $25(\mathrm{OH})-\mathrm{D} 3$ to be significantly higher in patients with high risk disease features with regard to medullary blast counts $\geq 5 \%$, cytogenetics, and IPSS-R (median serum level: 31,31 , and $30.5 \mathrm{ng} / \mathrm{mL}$, respectively) compared with patients with low risk disease features (median serum level: 16, 17, and $13 \mathrm{ng} / \mathrm{mL}$, respectively). In contrast to these findings, several studies conclude that low serum VD levels are associated with a poor outcome in cancer and hematological malignancies [21, 22]. These apparently conflicting findings might be due to the association of chronic inflammation and VD deficiency [10-12]. Experimental and clinical observations suggest that chronic inflammation within the tumor microenvironment is a hallmark of cancer progression [23]. Thus, low serum VD levels are considered to reflect advanced-stage disease. Regarding MDS, particularly lower-risk MDS are thought to be subjected to innate immune activation driving ineffective hematopoiesis and apoptosis [24] which might be reflected by markedly low serum 25(OH)-D3 concentrations in the realm of VD deficiency. Higher-risk MDS have been shown to have gained immune escape mechanisms substantially mediated by T-regulatory lymphocytes [24]. This immune attenuation might be reflected by a higher serum 25(OH)-D3 concentration as observed in our higher-risk MDS patients. The association between immune signaling and VD is further supported by the observation that in our patient cohort VD levels decrease while the NLR increases. NLR is a recognized tool for the assessment of the systemic inflammatory response and has been shown to be affected by VD [25]. Of clinical rel- evance is the association between MDS and autoimmune disorders that have been reported in several case report studies and long-term follow-up studies [26, 27]. Furthermore, two recent epidemiological studies showed that history of infection was significantly associated with a higher risk of developing MDS [27, 28].

The major limitation of our analysis is the lack of prospective data due to the fact that the assessment of serum $25(\mathrm{OH})$-D3 levels in patients with MDS was intended as real-world experience. Therefore, the serum 25(OH)-D3 level were measured once in every MDS patient regardless of therapy, and intraindividual time series of serum $25(\mathrm{OH})$-D3 levels were not assessed. We are aware that the serum $25(\mathrm{OH})-\mathrm{D} 3$ concentration is affected by various factors such as age, sex, nutrition, dietary supplements, season, and therapy $[19,29]$. Regarding age, sex, and nutritional status, our patients cohort represents a typical MDS patients cohort. Serum 25(OH)-D3 levels were measured irrespective of seasons and constitute an all-year mixture. About one third of patients were treated. Treatment data did not permit controlled analysis of the impact of therapy on serum 25(OH)-D3 levels. However, there were similar proportions of treated patients in the lower-risk disease subgroup (mainly erythropoietin) and higher-risk disease subgroup (mainly hypomethylating agents). From an epidemiological point of view, it is noteworthy that a comprehensive German health survey among 8,151 adults (18-79 years) revealed VD deficiency in a considerable proportion of adults aged 65-79 years (mean serum VD: 16.4 and $18 \mathrm{ng} / \mathrm{mL}$ in women and men, respectively) [30]. However, these data do not indicate an 
explanation for the differences of serum 25(OH)-D3 levels between lower-risk and higher-risk MDS patients. Therefore, we focused on this aspect and did not seek a comparison to an age-matched control group of healthy individuals.

In conclusion, we have demonstrated markedly reduced serum levels of 25(OH)-D3 in patients with MDS. Furthermore, we have shown that patients with lowerrisk MDS features clearly exhibit lower serum levels of $25(\mathrm{OH})-\mathrm{D} 3$ than patients with higher-risk disease characteristics. These intriguing findings might be ascribed to the innate immune deregulation in MDS and warrant further and deeper investigation in prospective MDS studies.

\section{Acknowledgements}

We thank Torsten Haferlach (MLL Munich) for providing cytogenetic data.

\section{Statement of Ethics}

Written informed consent in accordance with the Declaration of Helsinki was provided by all patients.

\section{Disclosure Statement}

The authors declare that there is no conflict of interest regarding the publication of this paper.

\section{Author Contributions}

C.M.-T., H.T., and K.S.G. designed the analysis and wrote the manuscript. C.M.-T., S.P., and K.S.G. collected and interpreted the data. H.T. performed the statistical analysis. M.R. reviewed the bone marrow slides. H.S. performed chemiluminescence immunoassay for measurement of serum levels of 25(OH)-D3. All authors reviewed and approved the final version of the manuscript.

\section{References}

1 Shastri A, Will B, Steidl U, Verma A. Stem and progenitor cell alterations in myelodysplastic syndromes. Blood. 2017 Mar;129(12):158694.

2 Jankowski M, Dyszkiewicz-Konwińska M, Magas M, Skorupski M, Gorecki G, Bukowska $D$, et al. Haematopoiesis: living in the shadow of stem cell differentiation. J Biol Regul Homeost Agents. 2018 Jan-Feb;32(1):1-6.

3 Bejar R, Stevenson K, Abdel-Wahab O, Galili N, Nilsson B, Garcia-Manero G, et al. Clinical effect of point mutations in myelodysplastic syndromes. N Engl J Med. 2011 Jun;364(26): 2496-506.

4 Papaemmanuil E, Gerstung M, Malcovati L, Tauro S, Gundem G, Van Loo P, et al.; Chronic Myeloid Disorders Working Group of the International Cancer Genome Consortium. Clinical and biological implications of driver mutations in myelodysplastic syndromes. Blood. 2013 Nov;122(22):3616-27.

5 Haferlach T, Nagata Y, Grossmann V, Okuno Y, Bacher U, Nagae G, et al. Landscape of genetic lesions in 944 patients with myelodysplastic syndromes. Leukemia. 2014 Feb;28(2): 241-7.

6 Sallman DA, Cluzeau T, Basiorka AA, List A. Unraveling the pathogenesis of MDS: the NLRP3 inflammasome and pyroptosis drive the MDS phenotype. Front Oncol. 2016 Jun; 6:151.

7 Chen X, Eksioglu EA, Zhou J, Zhang L, Djeu J, Fortenbery N, et al. Induction of myelodysplasia by myeloid-derived suppressor cells. J Clin Invest. 2013 Nov;123(11):4595-611.
8 Yan L, Zheng D, Xu RH. Critical role of tumor necrosis factor signaling in mesenchymal stem cell-based therapy for autoimmune and inflammatory diseases. Front Immunol. 2018 Jul;9:1658.

9 Lang PO, Aspinall R. Vitamin D status and the host resistance to infections: what it is currently (not) understood. Clin Ther. 2017 May; 39(5):930-45

10 Majewski K, Agier J, Kozłowska E, BrzezińskaBłaszczyk E. Status of cathelicidin IL-37, cytokine TNF, and vitamin D in patients with pulmonary tuberculosis. J Biol Regul Homeost Agents. 2018 Mar-Apr;32(2):321-5.

11 Fattizzo B, Zaninoni A, Giannotta JA, Binda F, Cortelezzi A, Barcellini W. Reduced 25-OH vitamin $\mathrm{D}$ in patients with autoimmune cytopenias, clinical correlations and literature review. Autoimmun Rev. 2016 Jul;15(7):770-5.

12 Delvin E, Souberbielle JC, Viard JP, Salle B. Role of vitamin $\mathrm{D}$ in acquired immune and autoimmune diseases. Crit Rev Clin Lab Sci. 2014 Aug;51(4):232-47.

13 Pandolfi F, Franza L, Mandolini C, Conti P. Immune modulation by vitamin $\mathrm{D}$ : special emphasis on its role in prevention and treatment of cancer. Clin Ther. 2017 May;39(5): 884-93.

14 Karlic H, Varga F. Impact of vitamin D metabolism on clinical epigenetics. Clin Epigenetics. 2011 Apr;2(1):55-61.

15 Arber DA, Orazi A, Hasserjian R, Thiele J, Borowitz MJ, Le Beau MM, et al. The 2016 revision to the World Health Organization classification of myeloid neoplasms and acute leukemia. Blood. 2016 May;127(20):2391-405.
16 Greenberg PL, Tuechler H, Schanz J, Sanz G, Garcia-Manero G, Solé F, et al. Revised international prognostic scoring system for myelodysplastic syndromes. Blood. 2012 Sep; 120(12):2454-65.

17 Schanz J, Tüchler H, Solé F, Mallo M, Luño E, Cervera J, et al. New comprehensive cytogenetic scoring system for primary myelodysplastic syndromes (MDS) and oligoblastic acute myeloid leukemia after MDS derived from an international database merge. J Clin Oncol. 2012 Mar;30(8):820-9.

18 R Core Team. R: A language and environment for statistical computing. R Foundation for Statistical Computing, Vienna, Austria, 2015. https://www.R-project.org/.

19 EFSA: Dietary reference values for vitamin D. EFSA J. 2016;14(10):4547.

20 Holick MF, Binkley NC, Bischoff-Ferrari HA, Gordon CM, Hanley DA, Heaney RP, et al. Evaluation, treatment, and prevention of vitamin D deficiency: an endocrine society clinical practice guideline. J Clin Endocrinol Metab. 2011;96:1911-30.

21 Duffy MJ, Murray A, Synnott NC, O'Donovan $\mathrm{N}$, Crown J. Vitamin D analogues: potential use in cancer treatment. Crit Rev Oncol Hematol. 2017 Apr;112:190-7.

22 Wang W, Li G, He X, Gao J, Wang R, Wang $\mathrm{Y}$, et al. Serum 25-hydroxyvitamin D levels and prognosis in hematological malignancies: a systematic review and meta-analysis. Cell Physiol Biochem. 2015;35(5):1999-2005. 
23 Berraondo P, Minute L, Ajona D, Corrales L, Melero I, Pio R. Innate immune mediators in cancer: between defense and resistance. Immunol Rev. 2016 Nov;274(1):290-306.

24 Gañán-Gómez I, Wei Y, Starczynowski DT, Colla S, Yang H, Cabrero-Calvo M, et al. Deregulation of innate immune and inflammatory signaling in myelodysplastic syndromes. Leukemia. 2015 Jul;29(7):1458-69.

25 Tabatabaeizadeh SA, Avan A, Bahrami A, Khodashenas E, Esmaeili H, Ferns GA, et al. High dose supplementation of vitamin D affects measures of systemic inflammation: reduction in high sensitivity C-reactive protein level and neutrophil to lymphocyte ratio (NLR) distribution. J Cell Biochem. 2017 Dec; 118(12):4317-22.
26 Ertz-Archambault N, Kosiorek H, Taylor GE, Kelemen K, Dueck A, Castro J, et al. Association of therapy for autoimmune disease with myelodysplastic syndromes and acute myeloid leukemia. JAMA Oncol. 2017 Jul;3(7): 936-43.

27 Kristinsson SY, Björkholm M, Hultcrantz M, Derolf AR, Landgren O, Goldin LR. Chronic immune stimulation might act as a trigger for the development of acute myeloid leukemia or myelodysplastic syndromes. J Clin Oncol. $2011 \mathrm{Jul} ; 29(21): 2897-903$.

28 Titmarsh GJ, McMullin MF, McShane CM, Clarke M, Engels EA, Anderson LA. Community-acquired infections and their association with myeloid malignancies. Cancer Epidemiol. 2014 Feb;38(1):56-61.
29 Ye CF, Pan YM, Zhou H. Regulation of vitamin D receptor and Genistein on bone metabolism in mouse osteoblasts and the molecular mechanism of osteoporosis. J Biol Regul Homeost Agents. 2018 May-Jun;32(3):497505.

30 Rabenberg M, Scheidt-Nave C, Busch MA, Rieckmann N, Hintzpeter B, Mensink GB. Vitamin D status among adults in Germanyresults from the German Health Interview and Examination Survey for Adults (DEGS1). BMC Public Health. 2015 Jul;15(1):641. 